\title{
Effects of low dose nicotine on attention and incidental learning in smokers and never-smokers Panoraia Andriopoulou*1 and Andrew Scholey²
}

Address: ${ }^{1}$ Mental Health Centre of Patras, Greece and ${ }^{2}$ University of Northumbria, UK

* Corresponding author

from International Society on Brain and Behaviour: 2nd International Congress on Brain and Behaviour Thessaloniki, Greece. 17-20 November 2005

Published: 28 February 2006

Annals of General Psychiatry 2006, 5(Suppl I):S218 doi:I0.1 186/I744-859X-5-SI-S2I8

\begin{abstract}
Background
Interest in the relation between cholinergic drugs and the cognitive function greatly increased with the neuropathological demonstrations that cholinergic receptors in the cerebral cortex are reduced in people who die with Alzheimer's disease and that this decrease correlates with the degree of cognitive impairment (Perry et al., 1978). Thus, several studies have investigated the effects of nicotinic and muscarinic agonists and antagonists on different aspects of attention and memory (Spilich et al., 1992, Jones et al., 1992).
\end{abstract}

\section{Materials and methods}

The present study employed a between participants, double-blind, placebo controlled, randomised design in order to look at the effects of nicotine on different aspects of attention and incidental learning. Thirty-six deprivedsmokers and 36 never-smokers were administered nicotine or placebo via a Nicorette Inhalator and underwent tasks which have previously been reported to be differentially sensitive to changes in cholinergic activity. These tasks involved incidental learning (Semantic Priming task), focused attention (Rapid Visual Information Processing task) and broadened attention (Water Jars task).

\section{Results}

The results of the present investigation did not reveal any significant effect of nicotine inhalation on either the RVIP task or the WJ task. However, there was a clear trend for both deprived-smokers and never-smokers, who received nicotine to perform better on the RVIP task than those who received placebo. In terms of the WJ task the present findings suggest that nicotine impaired deprived-smokers' performance mostly. Perhapst the most interesting finding arising from the current study is that nicotine signifi- cantly improved performance on the Smantic Priming task in both deprived-smokers and never-smokers.

\section{Discussion}

The results of the current study did not provide any firm evidence that nicotine enhances focused attention although the trend in the data clearly added some weight to the hypothesis of a cholinergic mechanism mediating focused attention. No definite conclusions can be drawn for the effects of the drug on broadened attention since the data were more ambiguous. Nevertheless, the study has shed some light on the effects of nicotine inhalation on incidental learning. Since nicotine improved performance on the Semantic Priming task, it could be suggested that the mechanism implicated in incidental learning resides in the cholinergic system. Based only upon the results of the current investigation though, it is clearly premature to draw any definite conclusions and further research is essential.

\section{References}

I. Jones GMM, Sahakian BJ, Warburton DM, Gray JA: Effects of acute subcutaneous nicotine on attention, information processing and short-term memory in Alzheimer's disease. Psychopharmacology 1992, 108:485-494.

2. Scholey AB, Micklethwaite K, Mitchell D, Moss MC, Wesnes KA: Nicotine and oxygen differentially affect performance on tasks of incidental learning and attention. Proceedings of the British Psychological Society 1999, 7:1 I0.

3. Perry E, Tomlinson BE, Blessed G, Bergmann K, Gibson PH, Perry RH: Correlation of cholinergic abnormalities with senile plaques and mental test scores in senile dementia. BMJ 1978, 2:1457-1459.

4. Spilich GJ, June L, Renner J: Cigarette smoking and cognitive performance. Journal of addiction 1992, 87:1313-1326. 